\title{
A Note on the Comparisons Among Coherent Systems
}

\author{
Mohammad Khanjari Sadegh* and Tahere Tavasolian \\ University of Birjand
}

\begin{abstract}
Using the concept of system signature introduced by Samaniego (1985), Kochar et al. (1999) compared the lifetimes of the systems in which the lifetimes of the components are independent and identically distributed (i.i.d.) random variables. Their results are extended to the systems with exchangeable components by Navarro et al. (2005). This paper gives some alternative proofs to obtain their results. Particularly in view of the hazard rate ordering, we compare two systems with different structures and components, which extends Theorem 8 in Navarro et al. (2005). We also compare two systems with different structures and components in view of the likelihood ratio ordering. Some illustrative examples are mentioned.
\end{abstract}

Keywords. Coherent systems; stochastic ordering; hazard rate ordering; likelihood ratio ordering; signatures.

\section{Introduction}

A system consist of $n$ components is said to be coherent if every component of the system is relevant and the system is monotone (see the definition and a thorough study of coherent systems in Barlow and Proschan, 1975). Let the nonnegative and absolutely continuous random variables $X_{1}, \ldots, X_{n}$ and $T=$ $\phi\left(X_{1}, \ldots, X_{n}\right)$ be the component lifetimes, and the lifetime of the system, respectively. We note that $T \in\left\{X_{(1: n)}, \ldots, X_{(n: n)}\right\}$ with probability one, where $X_{(i: n)}$ represents the $i$ th order statistics of component lifetimes, $i=$ $1,2, \ldots, n$. Regarding stochastic ordering of order statistics, several authors

* Corresponding author 
have obtained various results (see for example Navarro (2008), Ma (1998), Boland et al. (1998) and Boland et al. (1994)). Most of their results are useful for studying of the aging properties and reliability analysis of those coherent systems which have exactly an order statistic equivalent for their lifetimes. For example $T$ is $X_{(n-k+1: n)}$ when the system has a $k$-out-of$n$ structure. For some aging properties of such systems see e.g. Asadi and Bayramoglu (2005, 2006), Li and Zhao (2006) and Khanjari (2008). By using the concept of system's signature, a coherent system which has no $k$-out-of$n$ structure, can be studied via the $k$-out-of- $n$ systems. Hence this concept plays an important role in the reliability studies of the coherent systems. Samaniego (1985) defined the signature of a coherent system of order $n$ as the probability vector $\boldsymbol{p}=\left(p_{1}, \ldots, p_{n}\right)$ where

$$
p_{i}=P\left(T=X_{(i: n)}\right) \quad i=1, \ldots, n .
$$

When $X_{i}$ 's are independent and identically distributed (i.i.d.), he showed that a coherent system is in fact a mixture of $k$-out-of- $n$ systems with weights $p_{i}$ 's, that is

$$
P(T>t)=\sum_{i=1}^{n} p_{i} P\left(X_{(i: n)}>t\right) .
$$

Using this Kochar et al. (1999) obtained some ordering properties of coherent systems with i.i.d. components whose signatures are ordered. Navarro et al. (2005) extended their results to coherent systems with exchangeable components.

This article considers some alternative methods to obtain their results as well. We obtain the conditions that can be used to compare the systems with different structures and components in view of the hazard rate ordering and likelihood ratio ordering. These extend some results in Navarro et al. (2005).

\section{Main Results}

In this section we compare the lifetimes of the coherent systems in view of the usual stochastic ordering, hazard rate ordering and likelihood ratio ordering. For a comprehensive study on stochastic ordering see Shaked and Shanthikumar (1994). 
Let $X_{i}, T=\phi\left(X_{1}, \ldots, X_{n}\right), X_{(i: n)}$ and $p_{i}=P\left(T=X_{(i: n)}\right), i=1, \ldots, n$ are defined as in Section 1.

In the following theorem we show that two events $\left\{X_{(i: n)}>t\right\}$ and $\left\{X_{\pi_{1}}<\right.$ $\left.X_{\pi_{2}}<\cdots<X_{\pi_{n}}\right\}$ are independent, where $\left\{\pi_{1}, \ldots, \pi_{n}\right\}$ is a permutation of positive integers $\{1, \ldots, n\}$.

Theorem 1. Suppose $X_{1}, \ldots, X_{n}$ are nonnegative and absolutely continuous and exchangeable random variables, (that is the joint distribution of any permutation of $X_{i}$ 's is the same) and let $X_{(i: n)}, i=1, \ldots, n$ be ith order statistic and $\pi$ is an arbitary permutation of numbers $\{1, \ldots, n\}$. Then for $t \geqslant 0$ two events $\left\{X_{(i: n)}>t\right\}$ and $\left\{X_{\pi_{1}}<X_{\pi_{2}}<\cdots<X_{\pi_{n}}\right\}$ are independent.

Proof. In view of exchangeability assumption it is enough to show that $P\left(X_{1}<X_{2}<\cdots<X_{n}, X_{(i: n)}>t\right)=P\left(X_{(i: n)}>t\right) P\left(X_{1}<X_{2}<\cdots<X_{n}\right)$.

We have

$$
\begin{aligned}
P\left(X_{(i: n)}>t\right)= & P\left(X_{1}>t, \ldots, X_{n}>t\right)+n P\left(X_{1}<t, X_{2}>t, \ldots, X_{n}>t\right) \\
& +\left(\begin{array}{c}
n \\
2
\end{array}\right) P\left(X_{1}<t, X_{2}<t, X_{3}>t, \ldots, X_{n}>t\right)+\cdots \\
& +\left(\begin{array}{c}
n \\
i-1
\end{array}\right) P\left(X_{1}<t, X_{2}<t, \ldots, X_{i-1}<t, X_{i}>t\right. \\
& \left.X_{i+1}>t, \ldots, X_{n}>t\right) \\
= & n !\left\{P\left(t<X_{1}<X_{2}<\cdots<X_{n}\right)\right. \\
& +P\left(X_{1}<t<X_{2}<\cdots<X_{n}\right) \\
& +P\left(X_{1}<X_{2}<t<X_{3}<\cdots<X_{n}\right)+\cdots \\
& \left.+P\left(X_{1}<X_{2}<\cdots<X_{i-1}<t<X_{i}<\cdots<X_{n}\right)\right\} \\
= & n ! P\left(X_{1}<X_{2}<\cdots<X_{n}, X_{(i: n)}>t\right) .
\end{aligned}
$$

Therefore

$$
P\left(X_{1}<X_{2}<\cdots<X_{n}, X_{(i: n)}>t\right)=\frac{1}{n !} P\left(X_{(i: n)}>t\right) .
$$

So

$$
P\left(X_{1}<X_{2}<\cdots<X_{n}, X_{(i: n)}>t\right)=P\left(X_{1}<X_{2}<\cdots<X_{n}\right) P\left(X_{(i: n)}>t\right) .
$$

Hence the proof is completed. 
Theorem 1 is not true in non i.i.d. case. See the following example.

Example 1. Suppose $X_{1}$ and $X_{2}$ are two independent random variables which are uniformly distributed on $(0,1)$ and $(0,2)$, respectively. For $0<$ $t<1$ it is easy to see that $P\left(X_{1}<X_{2}\right)=\frac{3}{4}$ and $P\left(X_{(1: 2)}>t\right)=\frac{t^{2}-3 t+2}{2}$ whereas $P\left(t<X_{1}<X_{2}\right)=\frac{t^{2}-4 t+3}{4}$. That is for $\pi=\{1,2\}$ and $i=1$, two events $\left\{X_{(1: 2)}>t\right\}$ and $\left\{X_{1}<X_{2}\right\}$ are not independent.

In the following lemma we now establish a fundamental property of a system's signature.

Lemma 1. Let $X_{1}, \ldots, X_{n}$ be the exchangeable components lifetimes of a coherent system of order $n$, and let $T$ be the system lifetime. Then

$$
\begin{aligned}
P(T>t) & =\sum_{i=1}^{n} p_{i} P\left(X_{(i: n)}>t\right) \\
& =\sum_{i=1}^{n} p_{i} \sum_{j=0}^{i-1}\left(\begin{array}{c}
n \\
j
\end{array}\right) P\left(X_{1}<t, \ldots, X_{j}<t, X_{j+1}>t, \ldots, X_{n}>t\right) .
\end{aligned}
$$

Proof. By using Theorem 1 and in view of Lemma 1 in Navarro and Rychlik (2007) and Lemma 3.1 in Navarro et al. (2008), the proof of the lemma follows and hence is omitted.

Remark 1. In i.i.d. case when $X_{i}$ 's are independent and have a common distribution function $F$ and survival function $\bar{F}=1-F$, the equation (3) reduces to

$$
P(T>t)=\sum_{i=1}^{n} p_{i} P\left(X_{(i: n)}>t\right)=\sum_{i=1}^{n} p_{i} \sum_{j=0}^{i-1}\left(\begin{array}{l}
n \\
j
\end{array}\right)(F(t))^{j}(\bar{F}(t))^{n-j} .
$$

Now by using Lemma 1, we compare system lifetimes in view of the usual stochastic ordering. For two system lifetimes $T_{1}$ and $T_{2}, T_{1} \leqslant s t T_{2}$ (i.e. $T_{1}$ is smaller than $T_{2}$ in usual stochastic ordering) if and only if $\bar{F}_{1}(t)=$ $P\left(T_{1}>t\right) \leqslant \bar{F}_{2}(t)=P\left(T_{2}>t\right), t>0$. For two discrete distributions $\boldsymbol{p}_{1}=\left(p_{11}, \ldots, p_{1 n}\right)$ and $\boldsymbol{p}_{2}=\left(p_{21}, \ldots, p_{2 n}\right)$ on the integers $\{1, \ldots, n\}$, we write $\boldsymbol{p}_{1} \leqslant s t \boldsymbol{p}_{2}$ if and only if $\sum_{i=j}^{n} p_{1 i} \leqslant \sum_{i=j}^{n} p_{2 i}$ for $j=1, \ldots, n$.

Navarro et al. (2005) showed that if $\boldsymbol{p}_{1}$ and $\boldsymbol{p}_{2}$ be the signatures of two coherent systems of order $n$ with exchangeable components, also $T_{1}=$ 
$\phi_{1}\left(X_{1}, \ldots, X_{n}\right)$ and $T_{2}=\phi_{2}\left(Y_{1}, \ldots, Y_{n}\right)$ be their respective lifetimes such that $\left(X_{1}, \ldots, X_{n}\right) \leqslant s t\left(Y_{1}, \ldots, Y_{n}\right)$ and $\boldsymbol{p}_{1} \leqslant s t \boldsymbol{p}_{2}$ then $T_{1} \leqslant s t T_{2}$. In special case when $T_{1}=\phi_{1}\left(X_{1}, \ldots, X_{n}\right)$ and $T_{2}=\phi_{2}\left(X_{1}, \ldots, X_{n}\right)$ and $\boldsymbol{p}_{1} \leqslant s t \boldsymbol{p}_{2}$ then $T_{1} \leqslant s t T_{2}$. This extends Theorem 3 in Kochar et al. (1999) which is proved in i.i.d. case.

The following lemma compares two systems with different components.

Lemma 2. Let $\boldsymbol{p}_{1}$ and $\boldsymbol{p}_{2}$ be the signatures of the two systems of order $n$ with exchangeable components, and let $T_{1}=\phi_{1}\left(X_{1}, \ldots, X_{n}\right)$ and $T_{2}=$ $\phi_{2}\left(Y_{1}, \ldots, Y_{n}\right)$. If $X_{(i: n)} \leqslant s t Y_{(i: n)}, i=1, \ldots, n$ and $\boldsymbol{p}_{1} \leqslant s t \boldsymbol{p}_{2}$ then $T_{1} \leqslant s t T_{2}$.

Proof. It is known that if $\boldsymbol{p}_{1} \leqslant s t \boldsymbol{p}_{2}$ then $\sum_{i=1}^{n} g(i) p_{1 i} \leqslant \sum_{i=1}^{n} g(i) p_{2 i}$ for any increasing function $g(i)$. Let $g(i)=P\left(X_{(i: n)}>t\right)$ and since $P\left(X_{(i: n)}>\right.$ $t) \leqslant P\left(Y_{(i: n)}>t\right)$ we have

$$
\begin{aligned}
P\left(T_{1}>t\right)= & \sum_{i=1}^{n} p_{1 i} P\left(X_{(i: n)}>t\right) \leqslant \sum_{i=1}^{n} p_{2 i} P\left(X_{(i: n)}>t\right) \\
& \leqslant \sum_{i=1}^{n} p_{2 i} P\left(Y_{(i: n)}>t\right)=P\left(T_{2}>t\right)
\end{aligned}
$$

that is $T_{1} \leqslant s t T_{2}$.

Remark 2. Using a different approach the result of Theorem 1 has been proved in Lemma 1 of Navarro and Rychlik (2007). We also note that the conditions $X_{(i: n)} \leqslant s t Y_{(i: n)}, i=1, \ldots, n$ which are used in Lemma 2 are more weak than the condition $\left(X_{1}, \ldots, X_{n}\right) \leqslant s t\left(Y_{1}, \ldots, Y_{n}\right)$ which is used in Theorem 4 of Navarro et al. (2005).

We now examine the comparing of system lifetimes in view of the hazard rate ordering which is a stronger ordering than the usual stochastic ordering. $T_{1} \leqslant h r T_{2}$ (i.e. $T_{1}$ is smaller than $T_{2}$ in the hazard rate ordering) if and only if the ratio $\bar{F}_{2}(t) / \bar{F}_{1}(t)$ is nondecreasing for $t<F_{1}^{-1}(1)$, where $\bar{F}_{1}$ and $\bar{F}_{2}$ are survival functions of $T_{1}$ and $T_{2}$, respectively. It is equivalent to say that $r_{T_{1}}(t)=f_{1}(t) / \bar{F}_{1}(t) \geqslant r_{T_{2}}(t)=f_{2}(t) / \bar{F}_{2}(t)$ for all $t \geqslant 0$, where $r_{T_{i}}(t)$ and $f_{i}(t)$ are hazard function and probability density function of $T_{i}$, respectively. For two signatures $\boldsymbol{p}_{1}=\left(p_{11}, \ldots, p_{1 n}\right)$ and $\boldsymbol{p}_{2}=\left(p_{21}, \ldots, p_{2 n}\right)$ we say that $\boldsymbol{p}_{1} \leqslant_{h r} \boldsymbol{p}_{2}$ if and only if $\sum_{i=j}^{n} p_{2 i} / \sum_{i=j}^{n} p_{1 i}$ is nondecreasing in $j$.

Navarro et al. (2005) showed that if $\boldsymbol{p}_{1}$ and $\boldsymbol{p}_{2}$ be the signatures of the two systems of order $n$ with exchangeable components, and $T_{1}=\phi_{1}\left(X_{1}, \ldots, X_{n}\right)$ 
and $T_{2}=\phi_{2}\left(X_{1}, \ldots, X_{n}\right)$ be their respective lifetimes such that $X_{(i: n)} \leqslant_{h r}$ $X_{(i+1: n)}, i=1, \ldots, n-1$ and $\boldsymbol{p}_{1} \leqslant_{h r} \boldsymbol{p}_{2}$ then $T_{1} \leqslant_{h r} T_{2}$. We note that if the components of the systems are not common, this result may not hold even if the components of each system are i.i.d. and two systems have the same structure. See the following example.

Example 2. Suppose $T_{1}=\max \left\{X_{1}, \min \left(X_{2}, X_{3}\right)\right\}$ and $T_{2}=\max \left\{Y_{1}, \min \right.$ $\left.\left(Y_{2}, Y_{3}\right)\right\}$ where $X_{i}$ 's are independent with distribution function $F(x)=1-$ $\bar{F}(x)=x, 0<x<1$ and $Y_{i}$ 's are independent with distribution function $G(x)=1-\bar{G}(x)=x^{2}, 0<x<1$. We note that $F \leqslant_{h r} G$ or equivalently $X_{i} \leqslant h r Y_{j}$ for all $i$ and $j,(i, j=1,2,3)$. Hence using Theorem 4.4 in Boland et al. (1998) we have $X_{(i: 3)} \leqslant h r Y_{(i: 3)}$ for $i=1,2,3$. We also have $\boldsymbol{p}_{1}=\boldsymbol{p}_{2}=\left(0, \frac{2}{3}, \frac{1}{3}\right)$ and for $0<x<1$ it is easy to see that

$$
\begin{aligned}
& P\left(T_{1}>x\right)=1-F(x)\left(1-\bar{F}^{2}(x)\right)=x^{3}-2 x^{2}+1=(1-x)\left(1+x-x^{2}\right) . \\
& P\left(T_{2}>x\right)=1-G(x)\left(1-\bar{G}^{2}(x)\right)=x^{6}-2 x^{4}+1=\left(1-x^{2}\right)\left(1+x^{2}-x^{4}\right) .
\end{aligned}
$$

Although $P\left(T_{2}>x\right) \geqslant P\left(T_{1}>x\right)$ that is $T_{1} \leqslant s t T_{2}$ but $h(x)=\frac{P\left(T_{2}>x\right)}{P\left(T_{1}>x\right)}=$ $\frac{(1+x)\left(1+x^{2}-x^{4}\right)}{1+x-x^{2}}$ is not nondecreasing in $x$ as $h(1)=2<h(0.9)$, hence $T_{1} k_{h r}$ $T_{2}$.

Example 2 shows that the similar result given in Lemma 2, for comparing of the systems lifetimes in view of the usual stochastic ordering, is not hold in view of the hazard rate ordering. In this case we need to add a stronger condition which is given in the next lemma.

Lemma 3. Let $T_{1}=\phi_{1}\left(X_{1}, \ldots, X_{n}\right)$ and $T_{2}=\phi_{2}\left(Y_{1}, \ldots, Y_{n}\right)$ be two coherent systems with exchangeable components. If $X_{(i: n)} \leqslant h r Y_{(j: n)}$ for all $i, j=$ $1, \ldots, n$, then $T_{1} \leqslant{ }_{h r} T_{2}$.

Proof. Let $\boldsymbol{p}_{1}=\left(p_{11}, \ldots, p_{1 n}\right)$ and $\boldsymbol{p}_{2}=\left(p_{21}, \ldots, p_{2 n}\right)$ be the signatures of the systems and suppose $f_{(i: n)}(t)$ and $\bar{F}_{(i: n)}(t)$ are probability density and survival functions of $X_{(i: n)}$ and $g_{(i: n)}(t)$ and $\bar{G}_{(i: n)}(t)$ are those of $Y_{(i: n)}, i=$ $1, \ldots, n$. In view of Lemma 1 we have

$$
h(t)=\frac{P\left(T_{2}>t\right)}{P\left(T_{1}>t\right)}=\frac{\sum_{i=1}^{n} p_{2 i} P\left(Y_{(i: n)}>t\right)}{\sum_{i=1}^{n} p_{1 i} P\left(X_{(i: n)}>t\right)}=\frac{\sum_{i=1}^{n} p_{2 i} \bar{G}_{(i: n)}(t)}{\sum_{i=1}^{n} p_{1 i} \bar{F}_{(i: n)}(t)} .
$$

On differentiating both sides of the above equation with respect to $t$ we have $h^{\prime}(t)=\frac{\sum_{i=1}^{n} p_{1 i} f_{(i: n)}(t) \sum_{i=1}^{n} p_{2 i} \bar{G}_{(i: n)}(t)-\sum_{i=1}^{n} p_{1 i} \bar{F}_{(i: n)}(t) \sum_{i=1}^{n} p_{2 i} g_{(i: n)}(t)}{\left\{P\left(T_{1}>t\right)\right\}^{2}}$. 
The numerator can be written as follow

$$
\sum_{i=1}^{n} \sum_{j=1}^{n} p_{1 i} p_{2 j}\left\{f_{(i: n)}(t) \bar{G}_{(j: n)}(t)-\bar{F}_{(i: n)}(t) g_{(j: n)}(t)\right\}
$$

where all terms in summations are nonnegative as $\bar{G}_{(j: n)}(t) / \bar{F}_{(i: n)}(t)$ is nondecreasing in $t$ since $X_{(i: n)} \leqslant h_{r} Y_{(j: n)}$ for all $i, j=1, \ldots, n$. Therefore $h^{\prime}(t) \geqslant 0$ and hence $h(t)$ is nondecreasing that is $T_{1} \leqslant h r T_{2}$. This completes the proof of the lemma.

Remark 3. Lemma 3 extends Theorem 8 in Navarro et al. (2005). The structures of the systems are not needed to be the same. We also note that if $T_{1}=\phi\left(X_{1}, \ldots, X_{n}\right)$ and $T_{2}=\phi\left(Y_{1}, \ldots, Y_{n}\right)$ such that $\left(X_{1}, \ldots, X_{n}\right) \stackrel{\text { st }}{=}$ $\left(Y_{1}, \ldots, Y_{n}\right)$ then $\boldsymbol{p}_{1}=\boldsymbol{p}_{2}$ and hence $T_{1} \stackrel{\text { st }}{=} T_{2}$. Also if $X_{i}$ 's, are i.i.d. with distribution function $F$ and $Y_{i}$ 's, are i.i.d. with distribution function $G$ again $\boldsymbol{p}_{1}=\boldsymbol{p}_{2}$ and if $F \leqslant s t G$ then $T_{1} \leqslant s t T_{2}$. But as we have seen in Example $2, F \leqslant \leqslant_{h r} G$ dose not necessary imply that $T_{1} \leqslant h r T_{2}$, unless the conditions of Lemma 3 hold that is $X_{(i: n)} \leqslant h r Y_{(j: n)}$ for all $i$ and $j$ or equivalently $X_{(n: n)} \leqslant h r Y_{(1: n)}$. Also in Example 2, the conditions of Lemma 3 are not hold as $X_{(3: 3)} K_{h r} Y_{(1: 3)}$.

Finally we examine the comparison of system lifetimes in view of the likelihood ratio ordering which is a stronger ordering than the hazard rate ordering. $T_{1} \leqslant_{l r} T_{2}$ (i.e. $T_{1}$ is smaller than $T_{2}$ in likelihood ratio ordering) if and only if the ratio $f_{2}(x) / f_{1}(x)$ is nondecreasing in $x$, where $f_{1}$ and $f_{2}$ are the probability density functions of $T_{1}$ and $T_{2}$, respectively. It is well known that $T_{1} \leqslant{ }_{l r} T_{2}$ implies that $T_{1} \leqslant{ }_{h r} T_{2}$ and $T_{1} \leqslant{ }_{h r} T_{2}$ implies that $T_{1} \leqslant s t T_{2}$. For two signatures $\boldsymbol{p}_{1}=\left(p_{11}, \ldots, p_{1 n}\right)$ and $\boldsymbol{p}_{2}=\left(p_{21}, \ldots, p_{2 n}\right)$ we say $\boldsymbol{p}_{1} \leqslant_{l r} \boldsymbol{p}_{2}$ if and only if $p_{2 i} / p_{1 i}$ is nondecreasing in $i$.

Navarro et al. (2005) showed that if $\boldsymbol{p}_{1}$ and $\boldsymbol{p}_{2}$ be the signatures of the two systems of order $n$ with exchangeable components, and $T_{1}=\phi_{1}\left(X_{1}, \ldots, X_{n}\right)$ and $T_{2}=\phi_{2}\left(X_{1}, \ldots, X_{n}\right)$ be their respective lifetimes such that $X_{(i: n)} \leqslant_{l r}$ $X_{(i+1: n)}, i=1, \ldots, n-1$ and $\boldsymbol{p}_{1} \leq_{l r} \boldsymbol{p}_{2}$ then $T_{1} \leqslant_{l r} T_{2}$.

Remark 4. We note that the above result holds true if we replace $T_{2}=$ $\phi_{2}\left(X_{1}, \ldots, X_{n}\right)$ by $T_{2}=\phi_{2}\left(Y_{1}, \ldots, Y_{n}\right)$ when $\left(X_{1}, \ldots, X_{n}\right) \stackrel{\text { st }}{=}\left(Y_{1}, \ldots, Y_{n}\right)$. In this case the condition $X_{(i: n)} \leqslant_{l r} X_{(i+1: n)}$ is not required. But in general if the systems consists different components this result may not hold. See the following example. 
Example 3. Suppose $T_{1}=\min \left(X_{1}, X_{2}\right)$ and $T_{2}=\min \left(Y_{1}, Y_{2}\right)$ where $X_{i}$ 's are independent and uniformly distributed on $(0,1)$, and $Y_{i}$ 's are independent and have exponential distribution with mean 1 . For $0<t<1$ we have $P\left(T_{1}>t\right)=(1-t)^{2}$ and $P\left(T_{2}>t\right)=e^{-2 t}$. Hence the density functions of $T_{1}$ and $T_{2}$ are $f_{1}(t)=2(1-t), 0<t<1$ and $f_{2}(t)=2 e^{-2 t}, 0<t$. Although $\frac{P\left(T_{2}>t\right)}{P\left(T_{1}>t\right)}=\frac{e^{-2 t}}{(1-t)^{2}}$ is nondecreasing in $t$ that is $T_{1} \leqslant_{h r} T_{2}$ but $\frac{f_{2}(t)}{f_{1}(t)}=\frac{e^{-2 t}}{1-t}$ is decreasing on $\left(0, \frac{1}{2}\right)$ and is increasing on $\left(\frac{1}{2}, 1\right)$. Therefore $T_{1}{ }_{l r} T_{2}$.

It is well known that if $X_{i}$ 's are i.i.d. then $X_{(i: n)} \leqslant l r X_{(i+1: n)}$ for $i=$ $1, \ldots, n-1$. Boland et al. (1994) showed that if $X_{i}$ 's are independent but not necessary identically distributed then $X_{(i: n)} \leqslant h r X_{(i+1: n)}$ for $i=$ $1, \ldots, n-1$. For $n=2$ they also constructed a counterexample and showed that $X_{(1: 2)} \Varangle_{l r} X_{(2: 2)}$. Hence if $X_{i}^{\prime}$ 's are independent and not identically distributed then the above mentioned results may not hold.

We now compare two systems with different structures and components. By using similar argument given in the proof of Lemma 3, it can be easily shown that if $T_{1}=\phi_{1}\left(X_{1}, \ldots, X_{n}\right), T_{2}=\phi_{2}\left(Y_{1}, \ldots, Y_{n}\right)$ and $X_{(i: n)} \leqslant l r Y_{(j: n)}$ for all $i$ and $j(i, j=1, \ldots, n)$ then

$$
\frac{f_{2}(t)}{f_{1}(t)}=\frac{\sum_{i=1}^{n} p_{2 i} g_{(i: n)}(t)}{\sum_{i=1}^{n} p_{1 i} f_{(i: n)}(t)}
$$

is nondecreasing in $t$ as $g_{(i: n)}(t) / f_{(i: n)}(t)$ is nondecreasing in $t$ since $X_{(i: n)} \leqslant l r$ $Y_{(j: n)}$ for all $i$ and $j$. That is $T_{1} \leqslant_{l r} T_{2}$ regardless the systems signatures are ordered or not.

\section{Conclusion Remarks}

We considered the stochastic orderings among coherent systems of the same order and consisting of components with exchangeable lifetimes. The approach used here is different with that of given by Kochar et al. (1999) and by Navarro et al. (2005). Particularly in view of the hazard rate ordering, we compared two systems with different structures and components. We also compared two systems with different structures and components in view of the likelihood ratio ordering. The conditions that can be used to compare the systems with different structures and components, in view of the hazard rate ordering and likelihood ratio ordering, are obtained. These extend some 
results in Navarro et al. (2005).

\section{Acknowledgment}

The authors are grateful to the referees for their helpful comments and suggestions, which have improved the article.

\section{References}

Asadi, M. and Bayramoglu, I. (2005). A note on the mean residual life function of a parallel system. Communications in Statistics-Theory and Methods, 34, 475-484.

Asadi, M. and Bayramoglu, I. (2006). The mean residual life function of a $k$-out-of- $n$ structure at the system level. IEEE Transactions on Reliability, 55, 314-318.

Barlow, R.E. and Proschan, F. (1975). Statistical Theory of Reliability and Life Testing: Reliability Models. Holt, Rinehart \& Winston, New York.

Boland, P.J., Neweihi, E.E. and Proschan, F. (1994). Applications of the hazard rate ordering in reliability and order statistics. Journal of Applied Probability, 31, 180-192.

Boland, P.J., Shaked, M. and Shanthikumar, G. (1998). Stochastic Ordering of Order Statistics. Handbook of statistics N. Balakrishnan and C.R. Rao, editions. Vol. 16, 89-103.

Khanjari, S.M. (2008). Mean past and mean residual life functions of a parallel system with nonidentical components. Communications in Statistics, Theory and Methods, 37, 1134-1145.

Kochar, S., Mukherjee, H. and Samaniego, F.J. (1999). The signature of a coherent system and its application to comparisons among systems. Naval Research Logistics, 46, 507-523.

Li, X. and Zhao, P. (2006). Some aging properties of the residual life of $k$-out-of- $n$ systems. IEEE Trans. Rel., 55, 535-541.

Ma, C. (1998). Likelihood ratio ordering of order statistics. Journal of statistical Planning and Inference, 70, 255-261.

Navarro, J. (2008). Likelihood ratio ordering of order statistics, mixtures and systems. Joural of statistical Planning and Inference, 138, 1242-1257.

Navarro, J., Ruiz, J.M. and Sandoval, C.J. (2005). A note on comparisons among coherent systems with dependent components using signatures. Statistics \& Probability Letters, 72, 179-185. 
Navarro, J. and Rychlik, T. (2007). Reliability and expectation bounds for coherent systems with exchangeable components. Journal of Multivariate Analysis, 98, 102-113.

Navarro, J., Samaniego, F.J., Balakrishnan, N. and Bhattacharya, D. (2008). On the application and extension of system signatures in engineering reliability. Naval Research Logistics, 55, 313-327.

Samaniego, F.J. (1985). On closure of the IFR class under formation of coherent systems. IEEE Transactions on Reliability, 34, 69-72.

Shaked, M. and Shanthikumar, J.G. (1994). Stochastic Orders and their Applications. Academic Press, San Diego.

Mohammad Khanjari Sadegh

Department of Statistics, University of Birjand, Birjand, Iran.

email: mkhanjari@birjand.ac.ir

\section{Tahere Tavasolian}

Department of Statistics, University of Birjand, Birjand, Iran. 